\title{
Addendum for Ann Fam Med. 2021;19(5):405-410.
}

Ann Fam Med 2022;20:4. https://doi.org/10.1370/afm.2780.

Table 1 in: Kerley B, Tan L, Marshall D, O'Neill C, Bialachowski A, Pereira J. COVID-19 personal protective equipment in the home: navigating the complexity of donning and doffing. Ann Fam Med. 2021;19(5):405-410 has been amended.

The online version of the article reflects the changes in Table 1 described below.

The protocol described in our original article ${ }^{1}$ was developed in the very early months of the pandemic, in the spring of 2020. At that time, high emphasis was placed on the possibility of fomite transmission and droplet transmission (vs aerosol transmission) of COVID-19, and our original protocol reflected this. Both of these premises, however, have been called into question as the pandemic has evolved. Fomite transmission has been de-emphasized ${ }^{2,3}$ and many authors have theorized that airborne transmission via aerosol is more common than originally thought. ${ }^{4,5}$ The appearance of the Omicron variant of the virus has also introduced new considerations. These include the high transmissibility and short intubation period of the virus, a high false negative rate of rapid antigen testing, and (at least in the Province of Ontario,
Canada) the lack of availability of timely PCR testing due to high demands. These developments have implications for both the procedures in the protocol and the type of personal protective equipment (PPE) needing to be worn. In light of this, we have revised our protocol.

\section{References}

1. Kerley B, Tan L, Marshall D, O'Neill C, Bialachowski A, Pereira J. COVID-19 personal protective equipment in the home: navigating the complexity of donning and doffing. Ann Fam Med. 2021;19(5):405-410.

2. Onakpoya IJ, Heneghan CJ, Spencer EA, et al. SARS-CoV-2 and the role of fomite transmission: a systematic review. F1000Res. 2021;10:233. 10.12688/ f1000research.51590.3

3. Goldman, E. Exaggerated risk of transmission of COVID-19 by fomites. Lancet Infect Dis. 2020;20(8):892-893.10.1016/S1473-3099(20)30561-2

4. Tang JW, Bahnfleth WP, Bluyssen PM, et al. Dismantling myths on the airborne transmission of severe acute respiratory syndrome coronavirus-2 (SARS-CoV-2). J Hosp Infect. 2021;110:89-96. 10.1016/j.jhin.2020.12.022

5. Public Health Agency of Canada. Statement from the chief public health office of Canada. Published Nov 12, 2021. https://www.canada.ca/en/ public-health/news/2021/11/statement-from-the-chief-public-health-officer-ofcanada-on-november-12-2021.html 